\title{
Improving the retrieval of information from external sources
}

\author{
SUSAN T. DUMAIS \\ Bellcore, Morristown, New Jersey
}

\begin{abstract}
A major barrier to successful retrieval from external sources (e.g., electronic databases) is the tremendous variability in the words that people use to describe objects of interest. The fact that different authors use different words to describe essentially the same idea means that relevant objects will be missed; conversely, the fact that the same word can be used to refer to many different things means that irrelevant objects will be retrieved. We describe a statistical method called latent semantic indexing, which models the implicit higher order structure in the association of words and objects and improves retrieval performance by up to $30 \%$. Additional large performance improvements of $40 \%$ and $67 \%$ can be achieved through the use of differential term weighting and iterative retrieval methods.
\end{abstract}

Although much research in cognitive psychology has been devoted to the question of how people retrieve information from their own memories, much less work has been done on the issue of the retrieval of information from external sources such as other people, books, libraries, or electronic databases. One problem that is immediately evident in attempting to retrieve information from external sources is the mismatch between the searcher's language and that of the target information. How often have you looked in the index of a book or the library catalog and been unable to find what you wanted? This problem is not evident in traditional memory modeling, because memory probes are in the same language as the memory representation.

Most approaches to the retrieval of electronically available textual materials depend on a lexical match between words in users' requests and those in database objects. Typically only text objects that contain one or more words in common with those in the users' query are returned as relevant. Word-based retrieval systems like this are, however, far from ideal-many objects relevant to a users' query are missed, and many unrelated or irrelevant materials are retrieved. A particularly salient example of the failure to find relevant materials is reported by Blair and Maron (1985) in a study of a state-of-the-art on-line legal retrieval system. Two lawyers, with the aid of an expert search intermediary, searched the database for all materials relevant to a case they were litigating. The system contained the full text of 40,000 documents, corresponding to roughly 350,000 pages of text. The lawyers were asked to search until they thought they had found $75 \%$ of the relevant materials. The surprising result was that they found only $20 \%$ of the known relevant materials.

Correspondence should be addressed to Susan T. Dumais, Bellcore, 445 South St., Room 2L-371, Morristown, NJ 07962-1910.
We believe that fundamental characteristics of human verbal behavior underlie these retrieval difficulties. Furnas, Landauer, Gomez, and Dumais (1987), for example, have shown that people generate the same main keyword to describe well-known objects less than $20 \%$ of the time. Comparably poor agreement has been reported in studies of interindexer consistency by Tarr and Borko (1974), and in the generation of search terms by expert intermediaries (Fidel, 1985) or by novices (Bates, 1986). Because of the tremendous diversity in the words people use to describe the same object or concept (synonymy), requesters will often use different words from the author or indexer of the information, and relevant materials will be missed. Conversely, since the same word often has more than one meaning (polysemy), irrelevant materials will be retrieved.

Several methods have been developed by researchers and practitioners in information retrieval (library science) to help overcome the problem of variability in human word usage and improve retrieval performance. These methods have included the following: restricting the allowable indexing and retrieval vocabulary and training intermediaries to generate terms from these restricted vocabularies; hand-crafting domain-specific thesauri to provide synonyms for users' search terms; constructing explicit models of domain-relevant knowledge; and automatically clustering terms and documents. The rationale for restricted or controlled vocabularies is that they are by design relatively unambiguous. They have high costs, however, and marginal (if any) benefits compared with automatic indexing based on the full content of texts. The use of a thesaurus is intended to improve retrieval by expanding terms that are too specific. Unfortunately, this also has the unwanted effect of retrieving irrelevant information. Overall, one can expect small retrieval improvements for carefully constructed thesauri in limited domains. More standard artificial intelligence techniques 
for knowledge representation are also beginning to be used for information retrieval. Such methods are currently applicable to small, stable domains and have not been systematically compared with more standard methods.

Automatic statistical methods for analyzing the relationships among words and documents are promising and much more widely applicable. We have developed a method called latent semantic indexing (LSI), which attempts to overcome the problems of variability in word usage by automatically organizing objects into a semantic structure more appropriate for information retrieval (Deerwester, Dumais, Furnas, Landauer, \& Harshman, 1990; Dumais, Furnas, Landauer, \& Deerwester, 1988). The LSI method begins by viewing the words or terms contained in a document as incomplete and unreliable indicators of the content of the document. We assume that there is some underlying or "latent" structure in the pattern of word usage that is partially obscured by the variability of word choice. We use statistical techniques to estimate this latent structure and get rid of the obscuring "'noise."' In this model, the similarity of terms and documents is determined by the overall pattern of word usage in the entire collection, so that documents can be similar to each other, regardless of the precise words they contain. A description of terms, objects, and user queries based on the underlying latent semantic structure, rather than surface-level words, is used for representing and retrieving information. What this means from a user's perspective is that documents can be similar to a query even if they share no terms in common.

\section{Overview of Latent Semantic Indexing}

Theory. The particular latent semantic indexing analysis that we have tried involves singular value decomposition (SVD), a technique closely related to eigenvector decomposition and factor analysis (Cullum \& Willoughby, 1985; Forsythe, Malcolm, \& Moler, 1977). We take a large matrix of term to text-object association data and decompose it into a set-typically 50-150-of orthogonal factors from which the original matrix can be approximated by linear combination. More formally, any rectangular matrix, $X$-for example, a $t \times o$ matrix of terms and objects-can be decomposed into the product of three other matrices:

$$
\underset{i \times o}{X}=\underset{i \times r}{T_{0}} \cdot \underset{r \times r}{S_{0}} \cdot \underset{r \times o}{O_{0}^{\prime}},
$$

such that $T_{0}$ and $O_{0}$ have orthonormal columns, $S_{0}$ is diagonal, and $r$ is the rank of $X$. This is a so-called singular value decomposition of $X$, and it is unique up to certain row, column, and sign permutations.

If only the $k$ largest singular values of $S_{0}$ are kept along with their corresponding columns in the $T_{0}$ and $O_{0}$ matrices, and the rest deleted (yielding matrices $S, T$, and $O)$, the resulting matrix, $\hat{X}$, is the unique matrix of rank $k$ that is closest in the least squares sense to $X$ :

$$
\underset{t \times o}{X} \approx \underset{t \times o}{\hat{X}}=\underset{t \times k}{T} \cdot \underset{k \times k}{S} \cdot \underset{k \times o}{O^{\prime}} .
$$

The idea is that the $\hat{X}$ matrix, by containing only the first $k$ independent linear components of $X$, captures the major associational structure in the matrix and throws out noise. It is this reduced model, usually with $k=100$, that we use to approximate the term to text-object association data in $X$. Since the number of dimensions in the reduced model $(k)$ is much smaller than the number of unique terms $(t)$, minor differences in terminology are ignored. In this reduced model, the similarity of objects is determined by the overall pattern of term usage, so that objects can be near each other regardless of the precise words that are used to describe them, and their description depends on a kind of consensus of their term meanings, thus dampening the effects of polysemy. In particular, this means that text objects that share no words with a user's query may still be near it if that is consistent with the major patterns of word usage. We use the term semantic indexing to describe our method, because the reduced SVD representation captures the major associative relationships between terms and text objects.

One can also interpret the analysis performed by SVD geometrically. The location of the terms and objects in $k$-space is given by the row vectors from the $T$ and $O$ matrices, respectively. In this space, the cosine or dot product between vectors corresponds to their estimated similarity. The position of term (document) vectors in this space reflects the correlations in their usage across documents (terms). This can be contrasted with lexical word-matching methods in which words are treated as independent. Retrieval typically proceeds by using the terms in a query to identify a vector in the space, and all text objects are then ranked by their similarity to the query. Since both terms and objects are represented in the same space, queries can be formed with any combination of terms and objects, and any combination of terms and objects can be returned in response to a query.

Our analysis is unlike many factor-analytic applications, in that we make no attempt to interpret the underlying dimensions or factors, nor to rotate them to some intuitively meaningful orientation. The analysis does not require us to be able to describe the factors verbally, but merely to be able to represent terms, text objects, and queries in a way that escapes the unreliability, ambiguity, and redundancy of individual terms as descriptors.

The idea of aiding information retrieval by discovering latent proximity structure has several lines of precedence in the information science literature. Hierarchical classification analyses have sometimes been used for term and document clustering (Jardin \& van Rijsbergen, 1971; Sparck Jones, 1971). Factor analysis has also been explored previously for automatic indexing and retrieval (Atherton \& Borko, 1965; Baker, 1962; Borko \& Bernick, 1963; Ossorio, 1966). Koll (1979) has discussed many of the same ideas described above regarding concept-based information retrieval, but his system lacks the formal mathematical underpinnings provided by the singular value decomposition model. Our latent structure method differs from these approaches in several important ways: (1) it involves a high-dimensional representation, which allows one to better represent a wide range of semantic relations; (2) both terms and text objects are 
explicitly represented in the same space; and (3) objects can be retrieved directly from query terms.

Practice. We have applied LSI to several standard information-science test collections, for which queries and relevance assessments were available. The text objects in these collections are bibliographical citations (consisting of titles, authors, and the full text of document abstracts), or the full text of short articles. A set of user queries and relevance judgments (judgments about the relevance of every document in the collection to each query) is associated with each test collection. Table 1 gives a brief description and summarizes some characteristics of the datasets and queries used in our experiments. As noted above, the "documents" in these collections consisted of the full text of document abstracts or short articles.

Results were obtained for LSI and compared against word-based retrieval methods. Each document is indexed completely automatically, and each word occurring in more than one document and not on a stop list of common words is included in the LSI analysis. The LSI anal$y$ sis begins with a large term $x$ document matrix in which cell entries are a function of the frequency with which a given term occurs in a given document. An SVD is performed on this matrix, and the $k$ largest singular values and their corresponding left and right singular vectors are used for retrieval. Queries are automatically indexed by means of the same preprocessing as was used for indexing the original documents, and the query vector is placed at the weighted sum of its constituent term vectors. The cosine between the resulting query vector and each document vector is calculated. Documents are returned in decreasing order of cosine, and performance is evaluated on the basis of this list.

The performance of information-retrieval systems is often summarized in terms of two parameters: precision and recall. Recall is the proportion of all relevant documents in the collection that are retrieved by the system; precision is the proportion of relevant documents in the set returned to the user. Precision is calculated for several levels of recall, and averaged across queries. (A signaldetection analysis could also be applied to these data, as Swets [1963] and others have noted. This is typically not done in the information-retrieval context, because there can be millions of correct rejections but only a handful of relevant documents or hits.)

The results for the MED collection are shown in Figure 1. Precision is plotted as a function of recall for nine levels of recall (from .10 to .90). These data represent average data from the 30 queries available with the MED collection. These are typical precision-recall curves, with precision (proportion of relevant information) decreasing as recall (proportion of relevant information found) increases. The important thing to notice is the difference between the LSI and word-matching methods. A 90dimensional LSI representation results in roughly $30 \%$ better performance in the discrimination of relevant from irrelevant documents. Over all the test collections, LSI averaged about $20 \%$ better than word-based methods, with a range from being comparable to, to being $30 \%$ better than, that obtained with standard vector methods. (See Deerwester et al., 1990, for details of these evaluations.)

\section{Improving Performance}

Although LSI, in comparison with word-matching methods, improves retrieval, the overall level of performance is still far from perfect-especially at high levels of recall. There are several well-known techniques for improving performance in standard word-based retrieval systems. We applied many of these methods to our LSI representation. One of the most important and robust methods involves differential term weighting (Sparck Jones, 1972). Another method of improvement involves an iterative retrieval process based on users' judgments of relevant items-often referred to as relevance feedback (Salton \& Buckley, 1990). The LSI approach also involves choosing the number of dimensions for the reduced space.

Choosing the number of dimensions. Choosing the number of dimensions for the reduced dimensional representation is an interesting problem. Thus far, our choice has been determined simply by what works best. We believe that the dimension-reduction analysis removes much of the noise, but that to keep too few dimensions would cause a loss of important information. We suspect that the use of too few dimensions has been a deficiency

Table 1

Characteristics of Datasets

\begin{tabular}{lrrrrr}
\hline & MED & CISI & CRAN & TIME & ADI \\
\hline Number of documents & 1,033 & 1,460 & 1,400 & 425 & 82 \\
Number of terms (occurring in more than one document) & 5,831 & 5,743 & 4,486 & 10,337 & 374 \\
Number of queries & 30 & 35 & 225 & 83 & 35 \\
Average number of documents relevant to a query & 23 & 50 & 8 & 4 & 5 \\
Average number of terms per document & 50 & 45 & 56 & 190 & 16 \\
Average number of documents per term & 9 & 13 & 16 & 8 & 4 \\
Average number of terms per query & 10 & 8 & 9 & 8 & 5 \\
Percent nonzero entries & 0.86 & 0.88 & 1.10 & 1.80 & 4.38 \\
\hline
\end{tabular}

Note-MED $=$ document abstracts in biomedicine received from the National Library of Medicine. CISI $=$ document abstracts in library science and related areas published between 1969 and 1977 and extracted from Social Science Citation Index by the Institute for Scientific Information. CRAN = document abstracts in aeronautics and related areas originally used for tests at the Cranfield Institute of Technology in Bedford, England. TIME = articles from Time magazine's world news section in 1963. ADI = small test collection of document abstracts from library science and related areas. 
MED (Entropy) Precision-Recall Curve

Average over queries

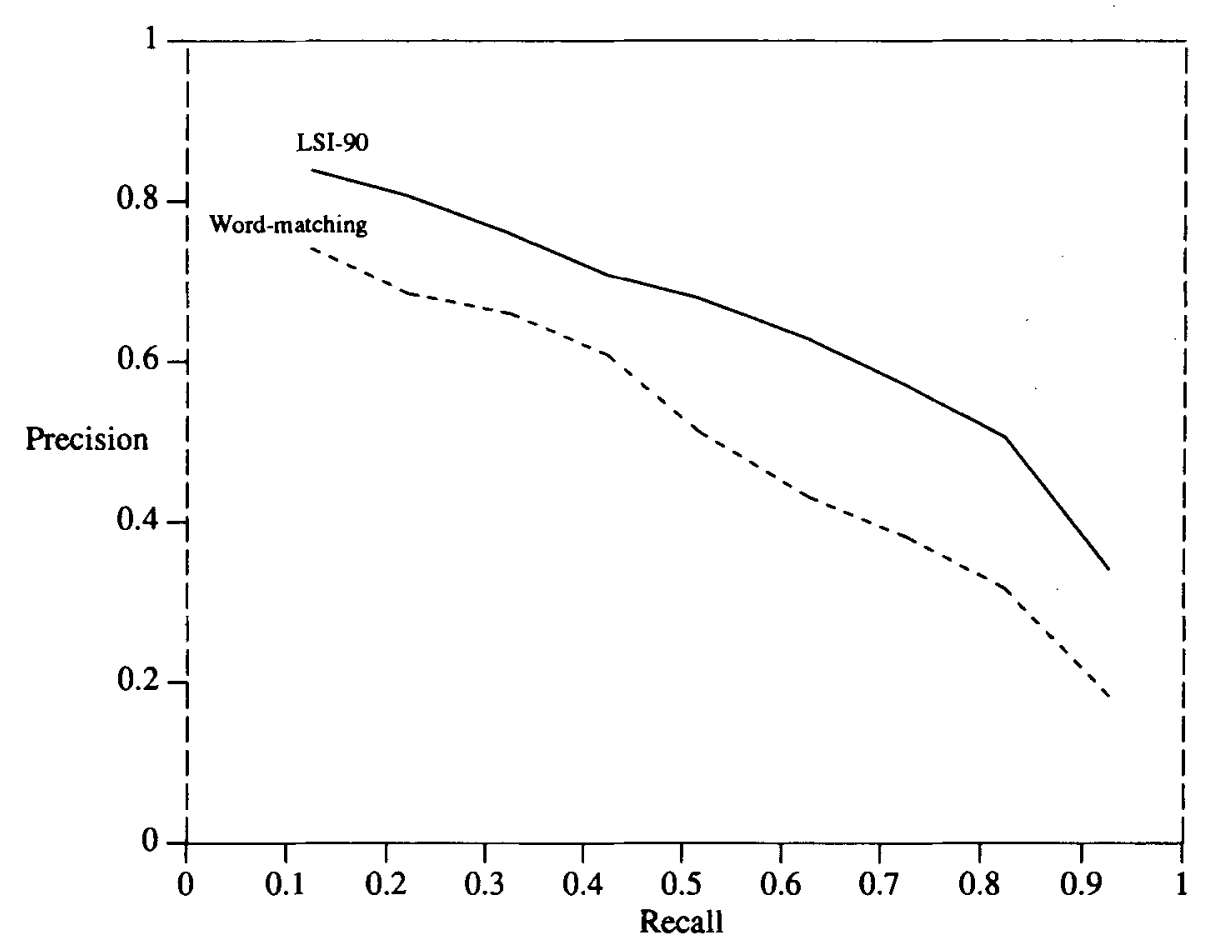

Figure 1. MED (entropy) precision recall.

of previous experiments in which techniques similar to SVD have been employed (Atherton \& Borko, 1965; Borko \& Bernick, 1963; Koll, 1979; Ossorio, 1966). Koll (1979), for example, used only seven dimensions to describe the relations among terms and documents. LSI retrieval performance was evaluated using a range of dimensions. In Figure 2, performance for the MED database is shown with different numbers of dimensions in the reduced LSI representation; performance consists of average precision over recall levels of $0.25,0.50$, and 0.75 .

The solid line in Figure 2 shows LSI performance as the number of dimensions in the reduced representation varies from 10 to 1,033 . The dashed line shows wordmatching performance. It is clear from this figure that performance improves considerably after 10 or 20 dimensions, peaks between 70 and 100 dimensions, and then begins to diminish slowly. This pattern of performance (initial large increase and slow decrease to word-based performance) is observed with other datasets as well. Theoretically, we expect the performance to increase only while the added dimensions continue to account for meaningful, as opposed to chance, co-occurrence. That LSI works well with a relatively small number of dimensions (small compared to the number of unique terms) shows that these dimensions are in fact capturing a major portion of the meaningful structure. As noted above, eventually performance must approach the level of performance attained by standard word-matching methods, because with sufficient parameters SVD will exactly reconstruct the original term by document matrix.

We have found that 100 -dimensional representations work well for these test collections. However, the number of dimensions needed to adequately capture the structure in other collections will probably depend on their breadth. Most of the test collections are relatively homogeneous, and 100 dimensions appears to be an adequate number to capture the major patterns of word usage across documents. In practice, the use of statistical heuristics for determining the dimensionality of an optimal representation will be important

Term weighting. One of the common and usually effective methods for improving retrieval performance is to give different terms different weights (Sparck Jones, 1972). The raw frequency of occurrence of a term in a document (i.e., the value of a cell in the raw term-document matrix) can be transformed. Such transformations normally have two components. Each term is assigned a global weight, indicating its overall importance in the collection as an indexing term. The same global weighting is applied to an entire row (term) of the term-docu- 
MED (LogEntropy)

Performance vs. number of dimensions

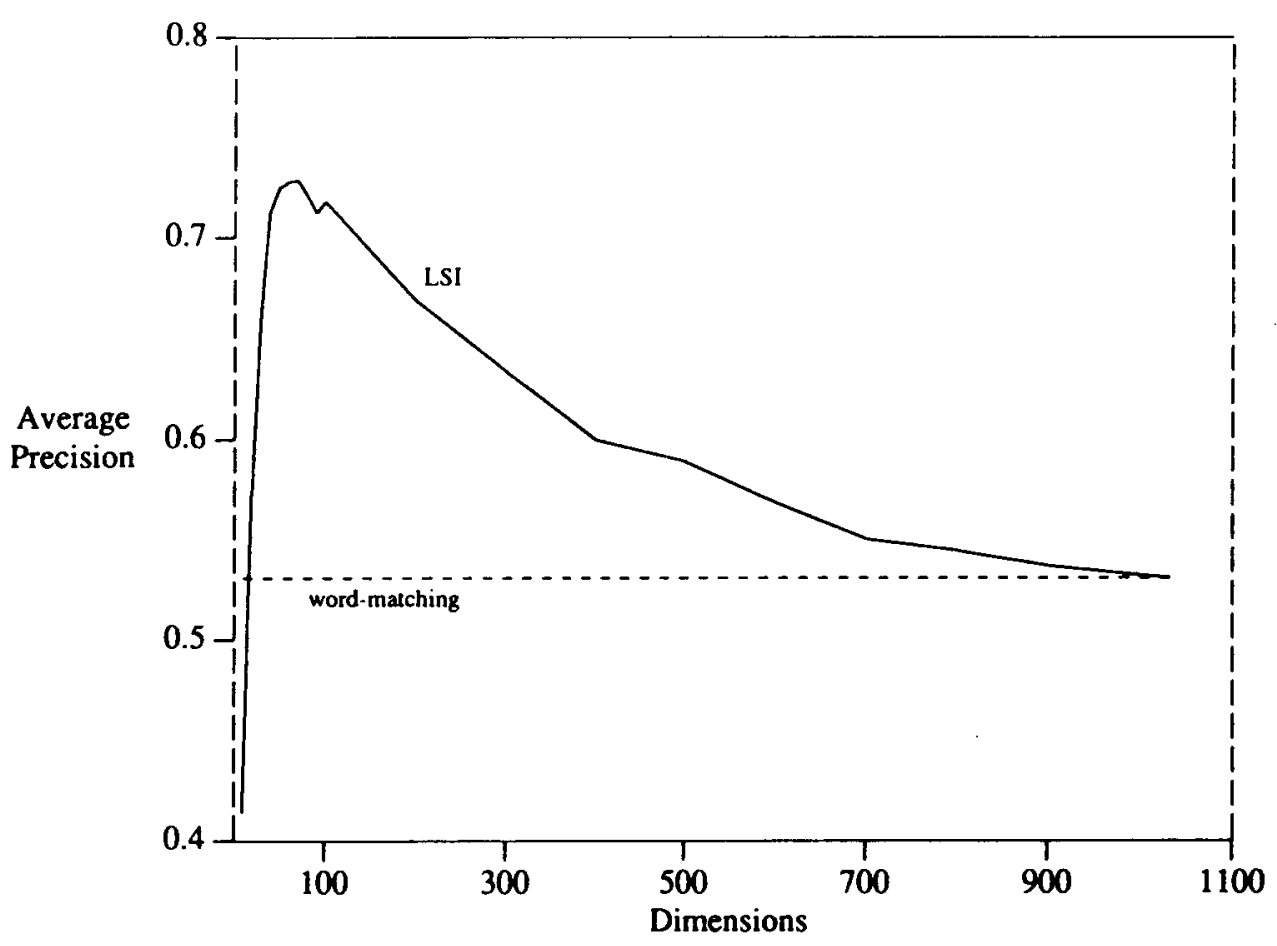

Figure 2. MED number of dimensions.

ment matrix. It is also possible to transform the term's frequency within a document; such a transformation is called a local weighting, and is applied to each cell in the matrix. The value for a term $i$ in a document $j$ is $L(i, j) \times G(i)$, where $L(i, j)$ is the local weighting for term $i$ in document $j$, and $G(i)$ is the term's global weighting.

Some popular local weightings include: term frequency, binary, and $\log ($ term frequency +1$)$. Term frequency is simply the frequency with which a given term appears in a given document. Binary weighting replaces any term frequency that is greater than or equal to 1 with 1 . $\log ($ term frequency +1$)$ takes the log of the raw term frequency, thus dampening effects of large differences in frequencies.

Four well-known global weightings are: Normal, Gfldf, Idf, and Entropy. Each is defined in terms of the term frequency $\left(t f_{i j}\right)$, which is the frequency of term $i$ in document $j$, the document frequency $\left(d f_{i}\right)$, which is the number of documents in which term $i$ occurs, and the global frequency $\left(g f_{i}\right)$, which is the total number of times that term $i$ occurs in the whole collection.

$\begin{array}{ll}\text { - Normal: } & \sqrt{\frac{1}{\sum_{j} f_{i j}^{2}}} \\ \text { - GfIdf: } & \frac{g f_{i}}{d f_{i}}\end{array}$

$$
\text { - Idf: } \quad \log _{2}\left(\frac{n \text { docs }}{d f_{i}}\right)+1 \text {, }
$$

where ndocs is the number of documents in the collection.

- 1 - entropy or noise:

$$
1-\sum_{j} \frac{p_{i j} \log \left(p_{i j}\right)}{\log (\text { ndocs })}
$$

where

$$
p_{i j}=\frac{t f_{i j}}{g f_{i}}
$$

and ndocs is the number of documents in the collection.

All of the global weighting schemes basically give less weight to terms that occur frequently or in many documents. Entropy is based on information-thenretic ideas and is the most sophisticated weighting scheme, taking the distribution of terms over documents into account.

We explored the effects of six different term weighting schemes in each of the test collections. We performed analyses using no global weighting (i.e., raw term frequency, $\left.t f_{i j}\right)$, combinations of the local weight $t f_{i j}$ and each of the four global weights discussed above (Gfldf, Idf, Entropy, and Normal), and one combination of a local log weight $\left[\log \left(t f_{i j}+1\right)\right]$ and a global entropy weight (LogEntropy). The original term $\times$ document matrix was transformed 
according to the relevant weighting scheme, and a reduced dimensional SVD was calculated and used for the analysis Sixty dimensions were used for the ADI collection, and 100 dimensions were used for the remaining collections. In all cases, query vectors were composed using the same weight that was used to transform the original matrix.

A summary of the term weighting experiments for the CRAN collection is presented in Figure 3. Precision is plotted as a function of recall for nine levels of recall (from .10 to .90 ). Data for each curve are averaged over 225 queries available with this collection. Differential term weighting has large effects on performance. Normalization and Gfldf are worse than no weighting, and Idf, Entropy, and LogEntropy all result in large improvements in performance, with LogEntropy being the best. Roughly comparable results are obtained with the other test collections as well. In all cases, LogEntropy results in the best retrieval performance, with an average advantage over raw term frequency of $40 \%$.

Relevance feedback. The idea behind relevance feedback is quite simple. Users are very unlikely to be able to specify their information needs adequately, especially given only one chance. With increases in computer speed, interactive or iterative searches are common, and users can reformulate queries in light of the system's response to previous queries (see, e.g., Oddy, 1977; Williams, 1984). There is surprisingly little experimental evidence to assess the success of users' successive attempts at query formulation. Another approach to query reformulation is to have the system automatically alter the query on the basis of user feedback about which documents are relevant to the initial request (Salton \& Buckley, 1990). This automatic system adaptation is what is usually meant by relevance feedback.

Simulations have shown that relevance feedback is quite effective (Salton \& Buckley, 1990; Stanfill \& Kahle, 1986). Systems can use information about which documents are relevant in many ways. Typically what is done is to increase the weight given to terms occurring in relevant documents and to decrease the weight of terms occurring in nonrelevant documents. Our tests using LSI have involved a method in which the initial query is replaced with the vector sum of the documents that the user has selected as relevant. We do not currently make use of negative information-for example, by moving the query away from documents that the user has indicated are irrelevant.

The document sets and user queries described in Table 1 were used in these experiments. We compared performance with the original user queries against two simulated cases of "feedback" and against a "centroid" query. Retrieval is first performed with the original query. Then this query is replaced by the first relevant document, the weighted average or centroid of the first three relevant documents, or the centroid of all relevant documents. The centroid condition represents the best performance that

Cranfield subset

Global weightings - LSI

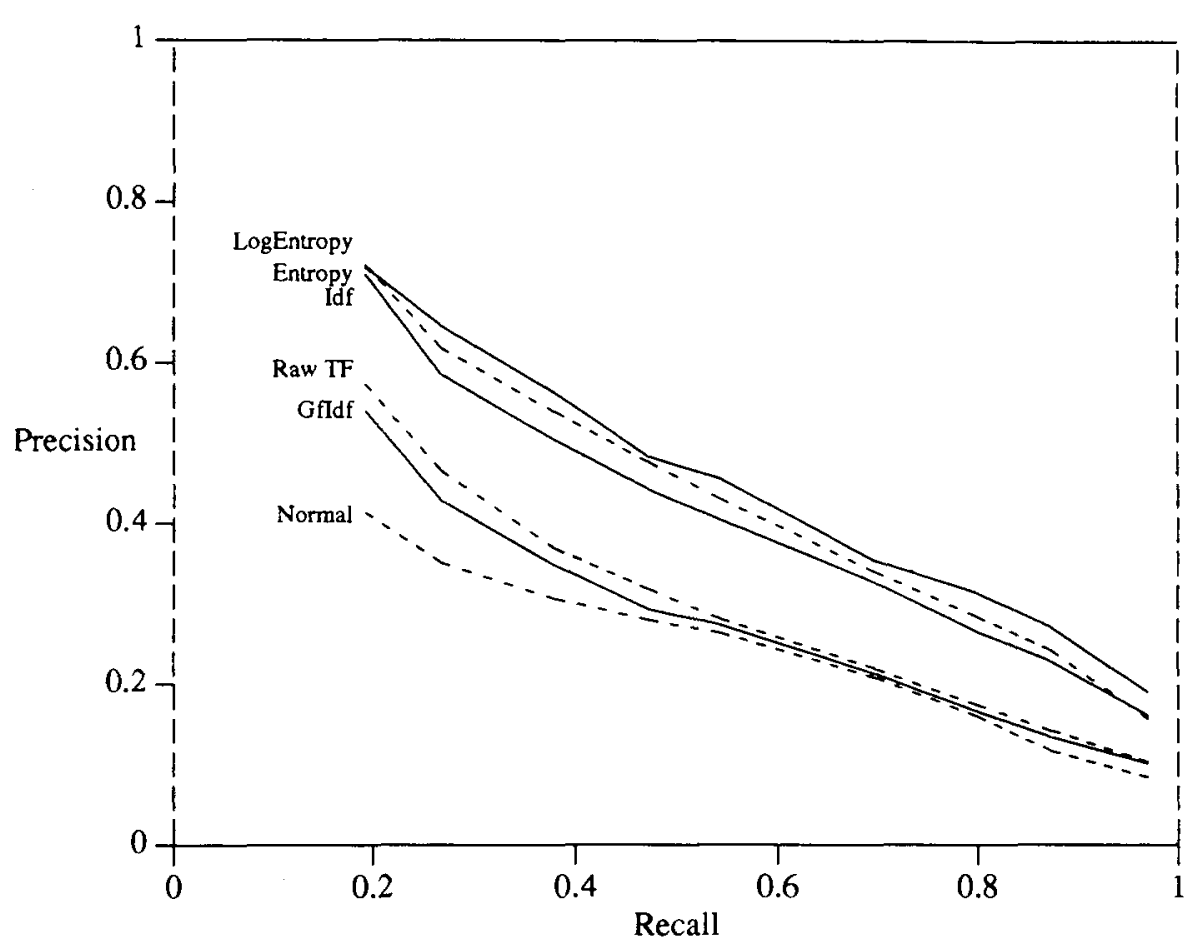

Figure 3. CRAN global term weightings. 
can be realized with a single-point query. Although this cannot be achieved in practice (except through many iterations), it serves as a useful upper bound on the performance that can be expected, given the LSI representation.

Large performance improvements were found for all but the MED dataset, where initial performance was already quite high. Performance improvements averaged $67 \%$ when the first three relevant documents were used as a query and $33 \%$ when the first document was used. These substantial performance improvements can be obtained with little cost to the user. A median of seven documents must be viewed in order for the user to find the first three relevant documents, and a median of one document must be seen in order for the user to find the first relevant document. Relevance feedback in which documents are used as queries imposes no added costs in terms of system computations. Since both terms and documents are represented as vectors in the same $k$-dimensional space, the formation of queries as combinations of terms (normal query), documents (relevance feedback), or both is straightforward.

The centroid query results in performance that is quite good (average precision of .80 or more) in all but one collection. This suggests that the LSI representation is generally adequate to describe the interrelations among terms and documents, but that users have difficulty in stating their requests in a way that leads to appropriate query placement. In the case of the CISI collection (where there are an average of 50 relevant documents), a single query does not seem to capture the appropriate regions of relevance (average precision of 7). We are now exploring a method for representing queries in a way that allows for multiple disjoint regions to be equally relevant to a query (Kane-Esrig, Casella, Streeter, \& Dumais, 1989).

How well relevance feedback (or other iterative methods) will work in practice is an empirical issue. We are now in the process of conducting an experiment in which users modify initial queries by means of: (1) rephrasing the original query; (2) relevance feedback based on user-selected relevant documents; (3) or a combination of both methods. (See Dumais \& Littman, 1990 , for a description of the interface and evaluation method.)

\section{Summary and Conclusions}

LSI is a modification of the vector-retrieval method that explicitly models the correlation of term usage across documents using a reduced dimensional SVD. The technique's tested performance ranged from being roughly comparable to that of standard vector methods, to being $30 \%$ better, apparently depending on the associative properties of the document set and the quality of the queries. These results demonstrate that there is useful information in the correlation of terms across documents, contrary to the assumptions behind many vector-model information retrieval approaches that treat terms as uncorrelated.

Performance in LSI-based retrieval can be improved by many of the same techniques that have been useful in standard vector-retrieval methods. In addition, varying the number of dimensions in the reduced SVD space influences performance. Performance increases dramatically over the first 100 dimensions, reaching a maximum and falling off slowly to reach the typically lower word-based level of performance. Idf and Entropy global term weighting improved performance by an average of $30 \%$, and improvements with the combination of a local log and a global entropy weighting (LogEntropy) were $40 \%$. In simulation experiments, relevance feedback using the first three relevant documents improved performance by an average of $67 \%$, and feedback using only the first relevant document improved performance by an average of $33 \%$. Since the first three relevant documents are found after a search through a median of only seven documents, this method offers the possibility of dramatic performance improvements with relatively little user effort. An experiment is now underway to evaluate the relevance feedback method in practice, and to compare it with other methods of query reformulation.

\section{REFERENCES}

Atherton, P., Borko, H. (1965). A test of factor-analytically derived automated classification methods (Rep. AIP-DRP 65-1).

BAKER, F. B. (1962). Information retrieval based on latent class analysis. Journal of the $A C M, 9,512-521$

BAtES, M. J. (1986). Subject access in online catalogs: A design model. Joumal of the American Society for Information Science, 37, 357-376.

Blair, D. C., \& MARON, M. E. (1985). An evaluation of retrieval effectiveness for a full-text document-retrieval system. Communications of the ACM, 28, 289-299.

Borko, H. \& Bernick, M. D. (1963). Automatic document classification. Journal of the $A C M, 10,151-162$.

Cullum, J. K., Willoughвy, R. A. (1985). Lanczos algorithms for large symmetric eigenvalue computations: Vol. I. Theory. Boston: Birkhauser.

Deerwester, S., Dumais, S. T., Furnas, G. W., Landauer, T. K., * Harshman, R. A. (1990). Indexing by latent semantic analysis. Joumal of the American Sociery for Information Science, 41, 391-407.

Dumais, S. T., Furnas, G. W., Landauer, T. K., De Derwester, S. (1988, May). Using latent semantic analysis to improve information retrieval. In $\mathrm{CHI}$ ' 88 Conference Proceedings: Human Factors in Computing Systems (pp. 281-285). New York: ACM.

Dumais, S. T., \& LitTman, M. L. (1990, April). InfoSearch: A program for iterative information retrieval using LSI [Poster]. $\mathrm{CHI}$ ' 90 Conference Proceedings: Human Factors in Computing Systems. New York: ACM.

FIDEL, R. (1985, October). Individual variability in online searching behavior. In C. A. Parkhurst (Ed.), ASIS '85: Proceedings of the ASIS 48th Annual Meeting (pp. 69-72). White Plains, NY: Knowledge Industry Publications.

Forsythe, G. E., Malcolm, M. A., Moler, C. B. (1977). Computer methods for mathematical computations. Englewood Cliffs, NJ: Prentice-Hall.

Furnas, G. W., Landauer, T. K., Gomez, L. M., a Dumais, S. T. (1987). The vocabulary problem in human-system communication. Communications of the ACM, 30, 964-971.

JARDIN, N., \& VAN RuSBERGen, C. J. (1971). The use of hierarchic clustering in information retrieval. Information Storage \& Retrieval, 7, 217-240.

Kane-Esrig, Y., Casella, G., Streeter, L. A., Dumais, S. T. (1989, August). Ranking documents for retrieval by modeling of a relevance density. In S. Boker (Ed.), Proceedings of the 12th IRIS (Information Systems Research Seminar in Scandinavia) (pp. 329-338). Aarhus, Denmark: Aarhus University. 
KolL, M. (1979). An approach to concept-based information retrieval. ACM SIGIR Forum, 13, 32-50.

ODDY, R. N. (1977). Information retrieval through man-machine dialogue. Joumal of Documentation, 33, 1-14.

Ossorio, P. G. (1966). Classification space: A multivariate procedure for automatic document indexing and retrieval. Multivariate Behavioral Research, 1, 479-524.

SALTON, G., \& BUCKLEY, C. (1990). Improving retrieval performance by relevance feedback. JASIS, 41, 288-297.

SPARCK JONES, K. (1971). Automatic keyword classification for information retrieval. London: Buttersworth.

SPARCK JONES, K. (1972). A statistical interpretation of term specificity and its applications in retrieval. Joumal of Documentation, 28, 11-21.
Stanfill, C., \& Kahle, B. (1986). Parallel free-text search on the connection machine system. Communications of the $A C M, 29$, 1229-1239.

SWETS, J. (1963). Information retrieval systems. Science, 141, 245250.

TARR, D., \& Borko, H. (1974, October). Factors influencing interindexer consistency. In P. Zunde (Ed.), Proceedings of the ASIS 37th Annual Meeting (pp. 50-55). Washington, DC: ASIS.

VoORHEES, E. (1985, June). The cluster hypothesis revisited. In SIGIR '85: Proceedings of the Eighth Annual International ACM SIGIR Conference on Research and Development in Information Retrieval (pp. 188-196). New York: ACM.

WILuAMS, M. D. (1984). What makes RABBIT run? Intemational Journal of Man-Machine Studies, 21, 333-352. 